\title{
Valores humanísticos importantes na formação do profissional médico: o que pensam os estudantes e professores
}

Important humanistic values in the formation of the medical professional: what students and teachers think

Valores humanísticos importantes en la formación de profesionales médicos: lo que piensan estudiantes y docentes

\author{
Adah Sophia Rodrigues Vieira \\ ORCID: https://orcid.org/0000-0002-8090-9606 \\ Universidade de Fortaleza, Brasil \\ E-mail: adahsophia0820@gmail.com \\ Victor Brasil Sá \\ ORCID: https://orcid.org/0000-0002-9305-6227 \\ Universidade de Fortaleza, Brasil \\ E-mail: victorbrasilsa@hotmail.com \\ Silvia Fernandes Ribeiro da Silva \\ ORCID: https://orcid.org/0000-0002-3223-5184 \\ Universidade de Fortaleza, Brasil \\ Centro Universitário Christus, Brasil \\ E-mail: silviafernandes@unifor.br \\ Rejane Maria Rodrigues de Abreu Vieira \\ ORCID: https://orcid.org/0000-0003-4475-6064 \\ Universidade de Fortaleza, Brasil \\ Universidade Estadual do Ceará, Brasil \\ E-mail: rejaneavieira@gmail.com \\ Sônia Leite da Silva \\ ORCID: https://orcid.org/0000-0003-2732-8543 \\ Universidade de Fortaleza, Brasil \\ Universidade Federal do Ceará, Brasil \\ E-mail: sonials@unifor.br
}

\begin{abstract}
Resumo
O objetivo deste estudo foi conhecer os valores humanísticos considerados pelos estudantes e professores de medicina como importantes na formação do discente. Estudo descritivo transversal. A população incluiu 203 alunos e 69 docentes do curso de medicina de uma universidade de Fortaleza. A coleta de dados foi realizada através de um questionário no Google Forms ${ }^{\circledR}$. O estudo foi aprovado pelo Comitê de Ética local. A média de idade entre os alunos foi $21,9 \pm 4,4$ anos, e entre os docentes foi 49,21 $\pm 1,3$ anos. A maioria dos avaliados era do sexo feminino. Entre os alunos, um total de 924 valores humanísticos foram contabilizados. Os quinze valores com maior frequência de citação foram agrupados em uma nuvem de palavras. Cerca de 20,1\% do total foi representado pelo conceito "empatia", seguido de "respeito" com 14,2\% e "humildade" com 7,9\%. Na avaliação dos docentes, a "empatia" (16\%) foi o valor mais citado, seguido por "ética" (11,7\%) e "respeito" (9\%). Valores como "compaixão", "honestidade", "resiliência" e "dedicação" foram citados de modo equilibrado por ambos os grupos. Empatia foi o valor humano mais citado, tanto pelos discentes como docentes, seguido por respeito, ética e humildade. Valores como compaixão, honestidade, resiliência foram também importantes. Esses valores devem ser incentivados durante todo o curso médico.
\end{abstract}

Palavras-chave: Valores sociais; Humanismo; Educação médica; Formação profissional; Ensino.

\begin{abstract}
The objective of this study is to know the humanistic values, considered by medical students and professors, as important in the education of students. Descriptive cross-sectional study. The population included 203 students and 69 professors from the medical course at a private university in Fortaleza. Data collection was performed using a questionnaire using Google Forms ${ }^{\circledR}$. The study was approved by the local Ethics Committee. The informed consent was completed and signed by participants. The average age among students was $21.9 \pm 4.4$ years, and among professors, it was $49.2 \pm 11.3$ years. Most of the evaluated individuals were female. Among the students, a total of 924 humanistic values were counted. The fifteen values with the highest citation frequency were grouped in a word cloud. About $20.1 \%$ of the total was represented by the concept "empathy", followed by "respect" with $14.2 \%$ and "humility" with $7.9 \%$. In the teachers' evaluation, "empathy" (16\%) was the most cited value, followed by "ethics" (11.7\%) and "respect" (9\%). Values such
\end{abstract}


as "compassion", "honesty", "resilience" and "dedication" were mentioned in a balanced way by both groups. Empathy was the most cited human value, both by students and teachers, followed by respect, ethics and humility. Values such as compassion, honesty, resilience were also important. These values must be encouraged throughout the medical course.

Keywords: Social values; Humanism; Medical education; Professional training; Teaching.

\section{Resumen}

El objetivo de este estudio fue conocer los valores humanísticos considerados por los estudiantes y profesores de medicina como importantes en la formación del estudiante. Estudio descriptivo transversal. La población estuvo compuesta por 203 estudiantes y 69 profesores de la carrera de medicina de una universidad de Fortaleza. La recolección de datos se realizó a través de un cuestionario en Google Forms ${ }^{\circledR}$. El estudio fue aprobado por el Comité de Ética local. La edad media de los estudiantes fue de 21,9 \pm 4,4 años y de los profesores de 49,21 \pm 1,3 años. La mayoría de los evaluados eran mujeres. Entre los estudiantes se registró un total de 924 valores humanísticos. Los quince valores con mayor frecuencia de citación se agruparon en una nube de palabras. Cerca del 20,1\% del total estuvo representado por el concepto "empatía", seguido de "respeto" con 14,2\% y "humildad" con 7,9\%. En la evaluación de los profesores, la "empatía" (16\%) fue el valor más citado, seguido de la "ética" $(11,7 \%)$ y el "respeto" (9\%). Valores como "compasión", "honestidad", "resiliencia" y "dedicación" fueron citados de manera equilibrada por ambos grupos. La empatía fue el valor humano más citado, tanto por estudiantes como por docentes, seguido del respeto, la ética y la humildad. Valores como la compasión, la honestidad, la resiliencia también fueron importantes. Estos valores deben ser incentivados a lo largo del curso de medicina.

Palabras-clave: Valores sociales; Humanismo; Educación médica; Formación profesional; Enseñanza.

\section{Introdução}

A medicina é uma das mais antigas profissões sendo considerada, por muitos, um ofício nobre, atrelado a um certo nível de sacerdócio. Esse estigma criado, muitas vezes, leva a um pensamento que exige uma demanda, uma personalidade compatível do praticante e não só um conhecimento científico. De fato, o exercício de tal ofício vai além de conhecimento técnico, puramente dito, transcendendo o saber científico, sendo necessários, também, a inteligência emocional, a capacidade de empatia e os valores éticos, uma vez que está lidando com a vida das pessoas (Ramos-Cerqueira \& Lima, 2002).

Independente da vertente de pensamento sobre o foco da área médica é imprescindível salientar a existência e a importância do lado mais humano da medicina, uma vez que ele é tão importante quanto a base de conhecimento puramente científico. Desse modo, assim como o conhecimento técnico, faz-se necessário educar e instruir os estudantes da área sobre a importância de conceitos básicos que rodeiam esse lado, como ética, moral e valores humanísticos de forma geral. De fato, a resolução de problemas e conflitos médicos, não se atrelam única e exclusivamente ao lado técnico, já que dilemas sociais fazem parte do cotidiano do profissional de saúde.

“A humanização da Medicina assume notável protagonismo na agenda dos educadores na Academia e dos gestores nos diversos Sistemas de Saúde. O motivo é claro: nos dias de hoje a Medicina tem de ser forçosamente humana se quer pautar-se pela qualidade e pela excelência. Humanizar a Medicina é, assim, além de uma obrigação educacional uma condição de sucesso para o profissional de saúde (Blasco, 2011).”

Logo, seguindo esse modelo de pensamento, em conjunto com o formato de ensino do Problem Based Learning (PBL), onde o aluno é confrontado com embates diversos e deve tentar desenvolver suas próprias soluções, esse trabalho visa propor uma forma ativa de ensino. Os dados adquiridos por meio da análise das respostas dos próprios alunos serão espelhados como resultado deste estudo e expostos a eles, a fim de que os próprios alunos façam a reflexão. Portanto, foi utilizada essa oportunidade para promover valores morais, utilizando o confronto com a realidade atual dos estudantes de medicina, visando, assim, maior humanização dessa área (Semerci, 2006).

“O primeiro passo que o médico - e o professor de Medicina - deve dar, se quer humanizar a Medicina, é admitir que, antes de tudo, se deve humanizar ele próprio. A responsabilidade primeira é toda dele, que deverá refletir e buscar recursos para integrar a técnica - atualizada e moderna - com o humanismo que a prática médica requer (Blasco, 2011).” 
O objetivo primário deste trabalho foi conhecer os valores humanísticos considerados pelos estudantes e professores de medicina como importantes para a formação do discente ao longo dos anos.

\section{Metodologia}

\subsection{Desenho e população de estudo}

Estudo descritivo, transversal e quantitativo. A população incluiu alunos dos ciclos básico $\left(1^{\circ}\right.$ e $2^{\circ}$ ano $)$ e clínico $\left(3^{\circ} \mathrm{e}\right.$ $4^{\circ}$ ano) do curso de medicina de uma universidade de Fortaleza-CE, além de membros do corpo docente do mesmo curso. O estudo foi realizado no período letivo de 2019 e 2020. Cada turma do ciclo básico, assim como do clínico, tem em média 90 alunos. Portanto, seriam cerca de 360 alunos por ciclo. Portanto, a amostra estimada é de 251 alunos. Em relação ao corpo docente, em que há um total de 157 professores, a amostra estimada é de 112 docentes.

$\mathrm{Na}$ área da saúde, os estudos de caráter descritivo ou observacional são bastante utilizados, são caracterizados como estudos que não interferem na trajetória do estudo, em que os pesquisadores atuam somente como observadores, registrando dados ou informações. (Kramer, 1988). A escolha desse método decorre, principalmente, do próprio instrumento escolhido (aplicação de questionário), o qual viabiliza uma coleta padronizada e sistematizada, em um dado período de tempo (Zangirolami-Raimundo, Echeimberg \& Leone, 2018).

Ademais, a escolha pela abordagem quantitativa foi fundamental, uma vez que a adoção de um delineamento observacional para o estudo indicou a necessidade de uma análise de caráter mais objetiva e imparcial. Apesar do tema subjetivo (valores humanos), o método quantitativo foi de maior significância para os objetivos do estudo, uma vez que a proposta inicial era de identificar e comparar os valores coletados de duas populações (Ferreira, 2015).

\subsection{Critérios de inclusão e exclusão}

Alunos regularmente matriculados no curso de Medicina da universidade, com idade igual ou superior a 17 anos e professores do referido curso, selecionados por meio de amostragem aleatória por conveniência. Foram excluídos todos aqueles que não concordaram em participar do estudo, além de alunos do Internato ( $5^{\circ}$ e $6^{\circ}$ ano), uma vez que o método de ensino é bastante diferente, em comparação aos primeiros quatro anos do curso.

\subsection{Metodologia proposta}

Após a leitura e assinatura do termo de consentimento livre e esclarecido (TCLE), os participantes aptos responderam a um questionário realizado via formulários do Google ${ }^{\circledR}$ composto por perguntas dissertativas. Além dos dados epidemiológicos e acadêmicos/profissionais, foram questionados quais os cinco principais valores humanísticos que o aluno e/ou professor consideravam essenciais na formação de um bom médico e, na opinião deles, se esses valores humanos estavam sendo estimulados durante o curso. Após a coleta, os dados passaram por uma análise e a partir deles foram obtidos os resultados a seguir expostos.

\subsection{Recrutamento da amostra}

O questionário foi divulgado para os alunos e professores utilizando os grupos de WhatsApp®. A divulgação foi realizada em mais de um momento, visando abranger o maior número possível de participantes.

\subsection{Análise dos dados}

Após a coleta, os dados foram analisados por meio do programa Excel ${ }^{\circ}$, sendo posteriormente enquadrados na "nuvem de palavras", por intermédio do programa Wordle ${ }^{\circ}$. A “nuvem de palavras" é uma ferramenta gratuita e de fácil acesso, sem 
patente própria, a qual possibilita a visualização de resultados de modo mais rápido e intuitivo (DePaolo \& Wilkinson, 2014; Ostapenko, 2020). Tal instrumento vem emergindo como uma importante ferramenta pedagógica, útil em diversas situações, tanto como objeto de estudo como complemento (Xie \& Lin, 2018).

\subsection{Aspectos éticos}

O estudo seguiu as Normas e Diretrizes estabelecidas pela Resolução no 466, de 12 de dezembro de 2012 do Conselho Nacional de Saúde/MS, atendendo às disposições preliminares, visando à ótica do indivíduo e da coletividade e aos quatros referenciais básicos da bioética: autonomia, não maleficência, beneficência e justiça. O Projeto foi aprovado pelo Comitê de Ética com o parecer de número 3.357.612 e todos os participantes responderam ao TCLE.

\section{Resultados}

Um total de 203 alunos responderam ao questionário com média de idade de 21,9 4,4 anos, sendo a maioria do sexo feminino (67,9\%). Dos 203 alunos, 96 (47,3\%) alunos eram do ciclo básico $\left(1^{\circ}\right.$ e $2^{\circ}$ ano) e $107(52,7 \%)$, do ciclo clínico $\left(3^{\circ} \mathrm{e}\right.$ $4^{\circ}$ ano). A amostra do corpo docente consistiu em 69 professores, com média de idade de 49,2 \pm 11,3 anos, sendo a maioria do sexo feminino $(66,7 \%)$. Sobre o tempo de magistério, a média foi de $11,4 \pm 10,9$ anos, e a maioria dos docentes era do ciclo básico $\left(1^{\circ} \mathrm{e} 2^{\circ}\right.$ ano).

Dentre os alunos, 189 (93,1\%) afirmaram haver estímulo adequado aos valores humanísticos durante a formação médica. De acordo com os valores humanísticos considerados pelos estudantes como importantes para a formação médica, foram contabilizados um total de 924 valores. Cerca de 20,1\% do total foi representado pelo conceito "empatia", seguido de "respeito" com 14,2\% e "humildade" com 7,9\%. Outros valores como "ética" (6,3\%), "honestidade" (5,2\%), "resiliência" (3,7\%) e "dedicação" (2,2\%), também foram lembrados, porém em menor proporção. Os 15 valores com maior frequência de citação foram agrupados em uma nuvem de palavras, mostrada na Figura 1, para a melhor visualização dos resultados, sendo o tamanho proporcional à frequência de cada valor.

Para fins de avaliação, as respostas foram divididas em ciclo básico e clínico, com o objetivo de entender a influência do nível de formação do aluno sobre os preceitos que regem o seu caráter moral e profissional. Sendo assim, levando-se em consideração o ciclo básico, foram obtidos 416 valores. Em comparação com o todo, a frequência de boa parte dos valores manteve a proporção, com exceção de "ética", que apresentou uma diminuição (4,8\%), e "honestidade", o qual apresentou um aumento na frequência $(7,2 \%)$. Além disso, atentando-se para os 15 valores humanísticos mais citados na Figura 2, observa-se a presença de "integridade", um conceito pouco valorizado em comparação com o todo. 
Figura 1: Nuvem de Palavras com os quinze principais valores humanísticos citados pelos estudantes.

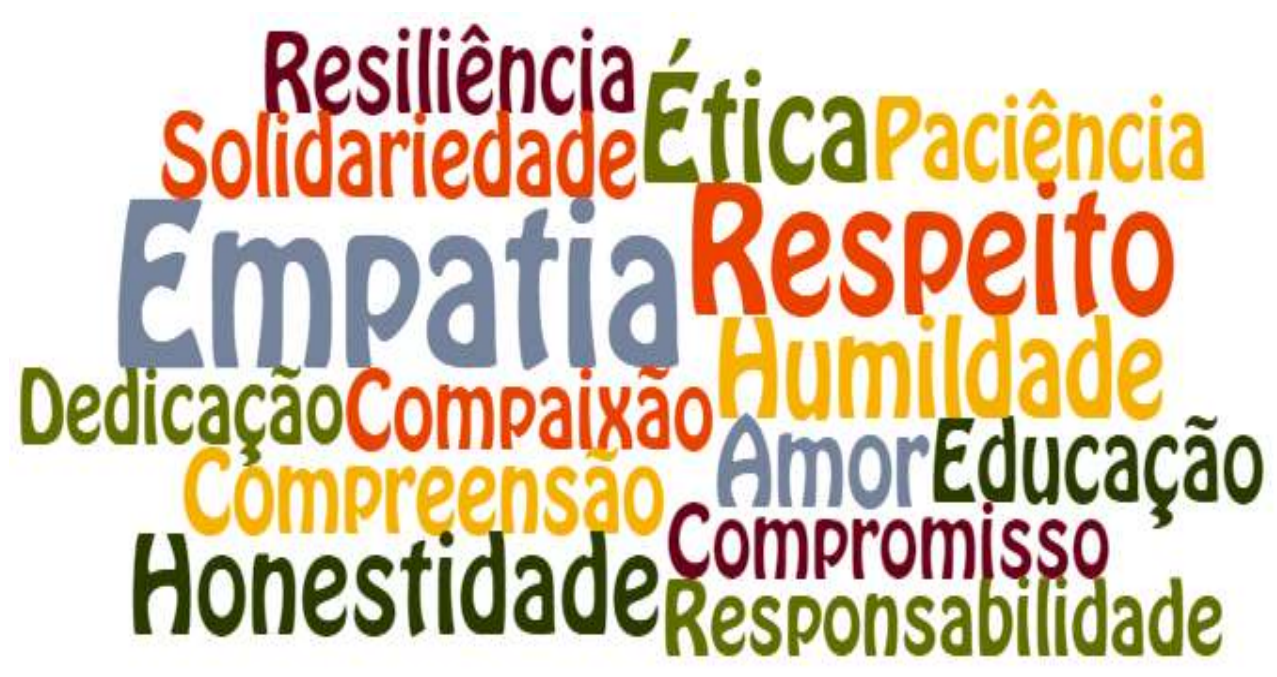

Fonte: Autores (2020).

Figura 2: Nuvem de Palavras com os quinze principais valores humanísticos citados pelos estudantes do ciclo básico.

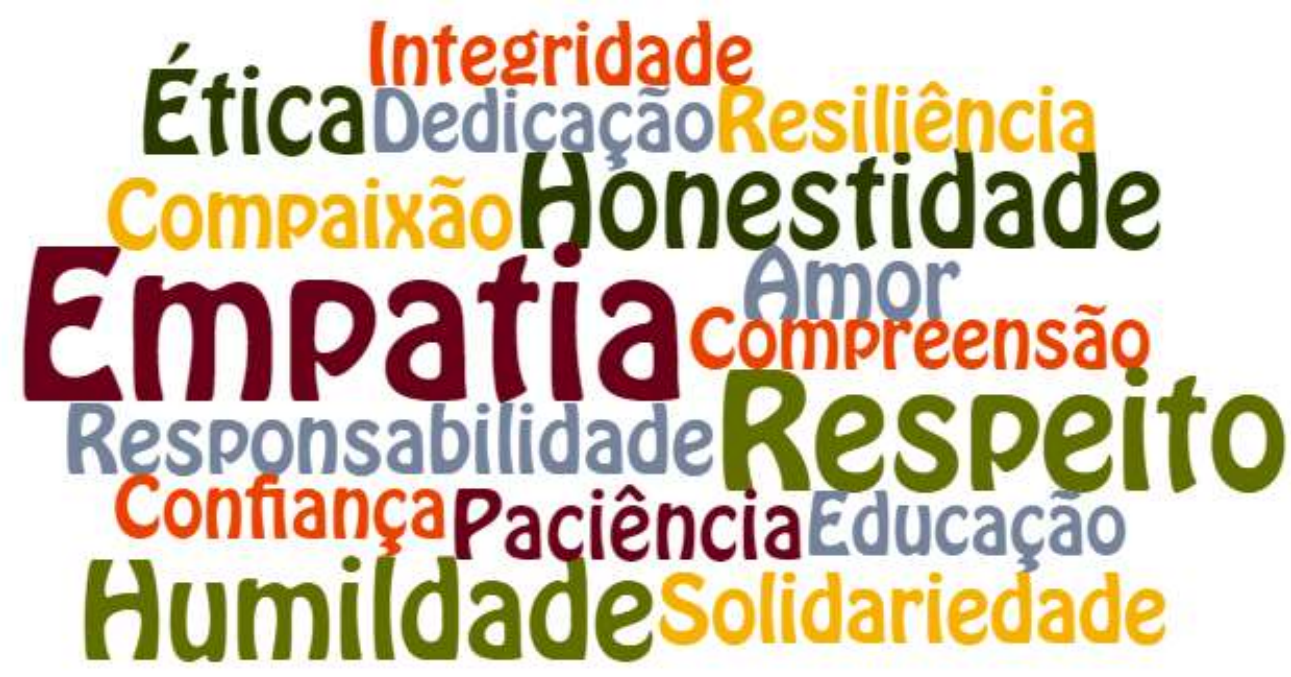

Fonte: Autores (2020).

Em relação ao ciclo clínico, foram coletados 508 valores humanísticos. À exemplo dos resultados do ciclo básico, a proporção dos valores manteve-se similar ao todo, exceto pela presença de "altruísmo" dentro dos quinze valores mais citados na Figura 3, em substituição ao valor “educação". 
Figura 3: Nuvem de Palavras com os quinze principais valores humanísticos citados pelos estudantes do ciclo clínico.

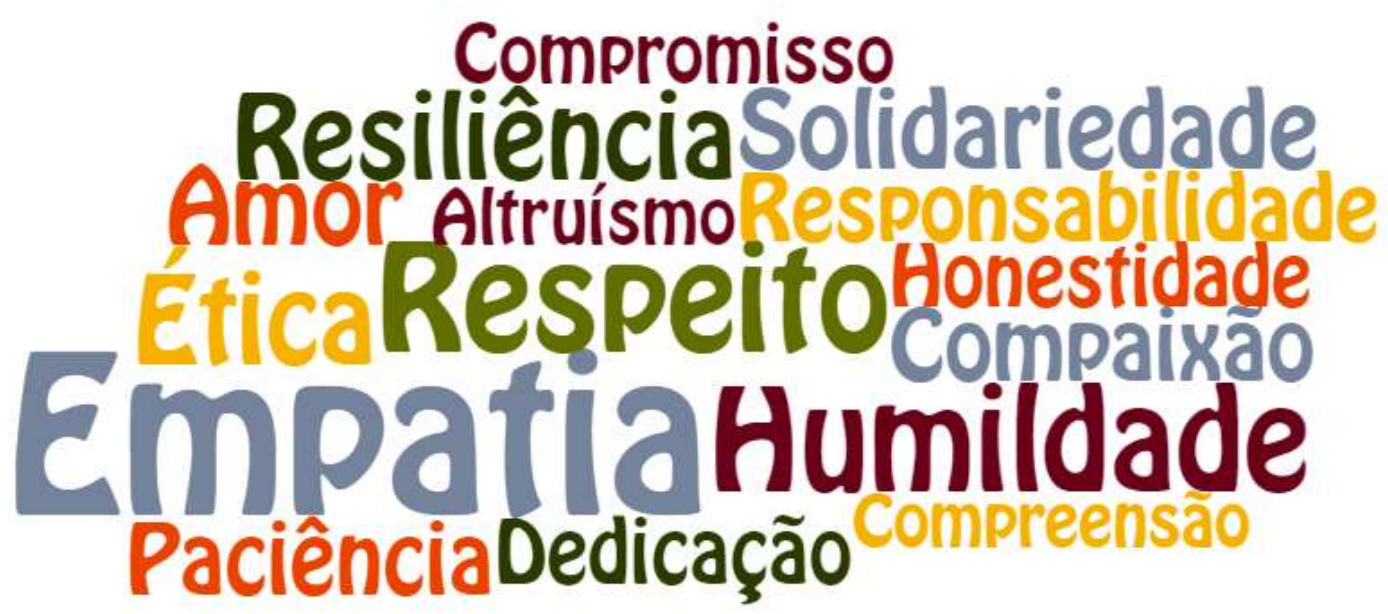

Fonte: Autores (2020)

Na avaliação dos docentes, a "empatia” (16\%) foi também o valor humanístico mais citado. Contudo, "ética” (11,7\%) adquire um papel mais relevante, em relação ao resultado dos estudantes, sendo o segundo preceito mais citado, enquanto que "respeito" foi o terceiro mais citado (9\%). Ademais, certos valores pouco citados pelos alunos, como "senso de justiça" (3,5\%), "escuta ativa" (3,2\%) e "bondade" (2,6\%) apresentam maior grau de frequência em comparação com os alunos. No geral, os quinze principais valores enumerados pelos professores pouco diferem dos citados pelos alunos, como evidenciado na Figura 4.

Figura 4: Nuvem de Palavras com os quinze principais valores humanísticos citados pelos professores.

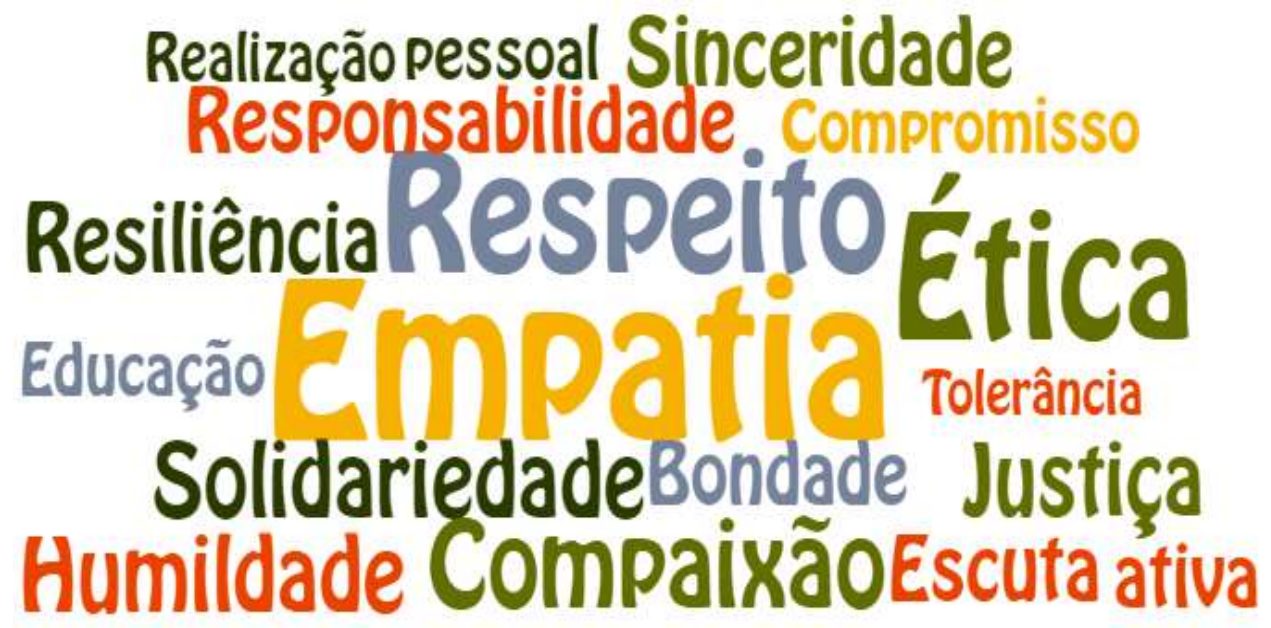

Fonte: Autores (2020)

\section{Discussão}

A inserção do humanismo no meio médico visa, sobretudo, evitar a progressão de uma crise social pré-existente, a qual apresenta, em seu cerne, um declínio de valores humanos que só poderia ser sanado quando for admitida a inegabilidade e importância desses princípios (Rosa et al., 2018). Entretanto, outros autores afirmam que o verdadeiro conhecimento moral somente é desenvolvido quando o próprio indivíduo é responsável pela sua conquista. Portanto, entende-se que o estudante é quem deve ter experiências que o façam compreender o que lhe é moralmente correto e ético (Piaget, 1996). 
Tendo em mente tal observação, destaca-se a ABP (Aprendizagem Baseada em Problemas) como método extremamente significativo, uma vez que ele estimula o pensamento crítico, motivando não somente a resolução de problemas, mas também o desenvolvimento de uma auto capacidade de julgamento em relação aos valores humanos importante para o futuro profissional (Semerci, 2006). Contudo, em associação a esse método, é necessário a presença de um outro fator bastante fundamental, que seria o corpo docente, o qual seria responsável pelos principais exemplos práticos aos quais alunos são expostos, uma vez que pode ser entendido que 'É necessário haver um clima sócio moral na instituição educativa e um 'estilo' do corpo docente que garantam um bom convívio entre professores e alunos" (Martín, 1998).

Todavia, essa associação entre a Medicina e a humanização ainda é um processo contínuo de construção e desenvolvimento, uma vez que, pela herança biológica e tecnicista da Medicina, ainda é muito comum julgar o ofício do médico como calculista e "frio", sendo tal pensamento compartilhando não somente por uma parcela da sociedade em si, mas também por parte da própria comunidade médica, que teve sua graduação regulada pelo paradigma tradicional. De acordo com a literatura, o tema de humanização na prática médica, apesar de considerado de suma importância, ainda é compreendido como um tema desnecessário (Rios et al., 2008).

Contudo, é importante destacar que o desenvolvimento do aprendizado humanizado dentro da Medicina encontra outros entraves além dos citados anteriormente. Um dos grandes obstáculos para fazer valer o currículo humanista na formação médica é a carga horária dos estudantes em formação, com um exaustivo consumo de tempo, fazendo com que o processo de ensino da ética seja prejudicado (Oji, 2020).

Sobre os resultados encontrados no presente estudo, observou-se a "Empatia" como o valor humanístico de maior importância, sendo selecionado pela maioria dos participantes discentes e docentes do estudo. Partindo da definição de empatia, interpreta-se tal valor como sendo o compartilhamento de emoções, em que uma pessoa consegue perceber e sentir a emoção da outra (Cecconello \& Koller, 2000). Portanto, entende-se que sua presença denota uma habilidade, assim como qualquer outra, que deve ser ensinada e praticada pelos estudantes de medicina. Uma das principais características da empatia é o seu caráter multidimensional, englobando aspectos subjetivos e emocionais, assim como dimensões objetivas e cognitivas (Provenzano et al., 2014). Sob tais conjecturas, é possível compreender que a elevada frequência desse valor entre os discentes e docentes devese, sobretudo, a sua presença durante toda a formação médica, especialmente no que diz respeito à relação médico-paciente, visto que é durante as consultas simuladas que o aluno consegue, por meio do ABP, pôr em prática essa habilidade humana (Di Blasi \& Kleijnen, 2003). Entretanto, a presença do valor empatia nos resultados encontrados, tanto no ciclo básico quanto no clínico, contraria a literatura, uma vez que, durante a formação médica, especialmente ao entrar no ciclo clínico, a maioria dos alunos adota posturas pouco empáticas, com progressiva queda dessa habilidade ao longo do tempo (Hojat et al., 2009).

"Respeito" foi o segundo valor humanístico mais citado pelo grupo de alunos, tanto em relação aos pacientes quanto aos pares (aluno-aluno). Este é um sentimento que muito repercute na interação humana e que traduz, de maneira importante, uma atitude mais humana, mais ligada às normas, e de grande valia no aprendizado do curso médico. Porém, a baixa frequência do valor "Ética" na amostra discente pode ser devido à complexidade que abrange o conceito, uma vez que inclui uma diversidade de incertezas e opiniões, geralmente baseadas em experiência pessoal e/ou profissional (Gerber \& Zagonel, 2013). Diferentemente dos alunos, os docentes consideraram a "Ética" como o segundo valor mais importante a ser guiado pelo bom médico, atribuindo uma maior relevância ao preceito. Indiscutivelmente, os valores éticos bem sedimentados são fundamentais para nortear a profissão médica (Siqueira, 2009). Esse fato pode ser devido ao maior grau de importância que o profissional atribui à ética, uma vez que a conduta do médico é pautada nos preceitos contidos no Código de Ética Médica (2018).

Além disso, os demais valores citados foram colocados de modo relativamente equilibrado entre os dois grupos de participantes, o que sugere uma padronização de pensamento. Entretanto, tal estabilização do pensamento vai contra os achados da literatura, uma vez que a maioria dos estudantes deveria apresentar mudanças no seu desenvolvimento moral, o que não parece 
ter ocorrido, quando comparados os resultados dos ciclos básico e clínico (Thomas, 2015). Contudo, tal padronização pode ser devido ao programa curricular do curso, sugerindo que, ao longo da formação individual, o ensino e a prática dos valores humanísticos apresentam uma natureza de maior estabilidade (Thomazi, Moreira \& Marco, 2012).

\section{Considerações Finais}

Medicina é uma profissão que demanda um grande grau de conhecimento científico, mas também exige um componente importante de inteligência emocional e valores humanos. Empatia foi o valor humano mais citado, tanto pelos discentes como por docentes do curso de medicina avaliado, seguido por respeito e ética, respectivamente entre os discentes e docentes. Valores humanísticos como humildade, solidariedade, honestidade, entre outros, foram citados de modo equilibrado entre os grupos, como essenciais na formação de um bom médico.

Em relação a estudos futuros, ressalta-se a relevância de identificar os valores humanos não somente durante os 4 primeiros anos da faculdade de Medicina, mas também com os dois últimos anos, sob um enfoque diferente, uma vez que esses estudantes estão mais próximos de se tornarem médicos profissionais, sendo, portanto, responsáveis por escolhas e decisões que devem ser pautadas tanto em conhecimento quanto em humanismo e moralidade.

\section{Agradecimentos}

A todos os participantes envolvidos na pesquisa e a todos que de alguma forma contribuíram para o desenvolvimento e publicação deste projeto.

\section{Referências}

Blasco, P. G. (2011). O Humanismo Médico: em Busca de uma Humanização Sustentável da Medicina. Revista Brasileira de Medicina, 68(1), 4-12. https://sobramfa.com.br/cientifico/wp-content/uploads/2014/10/2011_mai_o_humanismo_medico_humanizacao_sustentavel_da_medicina.pdf.

Cecconello, A. M. \& Koller, S. H. (2018). Competência social e empatia: um estudo sobre resiliência com crianças em situação de pobreza. Estudos de Psicologia (Natal), 5(1), 71-93. https://doi.org/10.1590/S1413-294X2000000100005.

Conselho Federal de Medicina (Brasil). Resolução CFM n 2.217, de 27 de setembro de 2018, modificada pelas Resoluções CFM n $2.222 / 2018$ e 2.226/2019. Diário Oficial da União, Seção 1.

DePaolo, C. A. \& Wilkinson, K. (2014). Get Your Head into the Clouds: Using Word Clouds for Analyzing Qualitative Assessment Data. TechTrends, 58(3), 38-44. https://doi.org/10.1007/s11528-014-0750-9.

Di Blasi, Z. \& Kleijnen, J. (2003). Context effects. Powerful therapies or methodological bias? Evaluation \& the Health Professions, 26(2), 166-79. https://doi.org/10.1177/0163278703026002003.

Ferreira, C. A. L. (2015). Pesquisa quantitativa e qualitativa: perspectivas para o campo da educação. Revista Mosaico, 8(2), 173-182. http://dx.doi.org/10.18224/mos.v8i2.4424.

Gerber, V. K. Q. \& Zagonel I. P. S. (2013). A ética no ensino superior na área da saúde: uma revisão integrativa. Revista Bioética, 21(1), 168-178. https://revistabioetica.cfm.org.br/index.php/revista_bioetica/article/download/795/869.

Hojat, M. et al. (2009). The Devil is in the Third Year: A Longitudinal Study of Erosion of Empathy in Medical School. Academic Medicine, 84(9), 1182-1191. https://doi.org/10.1097/acm.0b013e3181b17e55.

Kramer, M.S. (1988). Clinical Epidemiology and Biostatistics. Berlin: Springer-Verlag.

Martín, M. M. (1998). El contrato moral del profesorado: Condiciones para una nueva escuela. Bilbao: Editorial Desclée de Brouwer.

Oji, C. (2020). Human Values in Medicine-From Core Human Values (Universal Values) Perspective. Open Journal of Social Sciences, 8(4), 363-375. https://doi.org/10.4236/jss.2020.84026.

Ostapenko, R. I. (2020). "Word cloud" as a graphical supplement to a scientific article (Editor-in-chief's column). Economic consultant, 32(4), 4. http://dx.doi.org/10.46224/ecoc.2020.4.1.

Piaget, J. (1996). Os procedimentos da educação moral (1a ed.), São Paulo: Casa do Psicólogo.

Provenzano, B. C. et al. (2014). A empatia médica e a graduação em medicina. Revista HUPE, 13(4), 19-25. https://doi.org/10.12957/rhupe.2014.13941. 
Research, Society and Development, v. 11, n. 2, e31811225791, 2022 (CC BY 4.0) | ISSN 2525-3409 | DOI: http://dx.doi.org/10.33448/rsd-v11i2.25791

Ramos-Cerqueira, A. T. A. \& Lima, M. C. P. (2002). A Formação da Identidade do Médico: Implicações para o Ensino de Graduação em Medicina. Interface (Botucatu), 6(11), 107-106. https://doi.org/10.1590/S1414-32832002000200008.

Rios, I. C. et al. (2008) A integração das disciplinas de humanidades médicas na Faculdade de Medicina da USP - um caminho para o ensino. Revista Brasileira de Educação Médica, 32(1): 112-121. https://doi.org/10.1590/S0100-55022008000100015.

Rosa, N. V. D. L. et al. (2018). Reflexiones y avances en innovación educativa (1a ed.), Xalapa: RED Iberoamericana de Academias de Investigación AC.

Semerci, N. (2006). The effect of problem-based learning on the critical thinking of students in the Intellectual and Ethical Development Unit. Social Behavior and Personality: An international journal, 34(9), 1127-1136. https://doi.org/10.2224/sbp.2006.34.9.1127.

Siqueira, J. E. (2009). Ensino de ética no curso de medicina. O Mundo da Saúde, 33(1), 8-20. https://revistamundodasaude.emnuvens.com.br/mundodasaude/article/view/778/718.

Thomas, B. (2015). Moral and human values in medical education. Journal of Indian Society of Periodontology, 19(5), 486. https://dx.doi.org/10.4103\%2F0972124X.167209.

Thomazi, L. Moreira, F. G. \& Marco, M. F. (2014). Avaliação da evolução da empatia em alunos do quarto ano da graduação em medicina da Unifesp em 2012. Revista Brasileira de Educação Médica, 38(1), 87-93. https://doi.org/10.1590/S0100-55022014000100012.

Xie, Y. \& Lin, S-Y. (2018). Using word clouds to support students' knowledge integration from online inquiry: an investigation of the process and outcome. Interactive Learning Environments, 27(4), 478-496. https://doi.org/10.1080/10494820.2018.1484774.

Zangirolami-Raimundo, J. Echeimberg J.O. \& Leone, C. (2018). Research methodology topics: Cross-sectional studies. Journal of Human Growth and Development, 28(3), 356-360. http://dx.doi.org/10.7322/jhgd.152198. 